\title{
Three new species of the leafhopper genus Tautoneura Anufriev (Hemiptera, Cicadellidae, Typhlocybinae) from China
}

\author{
Yuehua Song ${ }^{1,2, \dagger}$, Zizhong Li ${ }^{2, \ddagger}$, Kangning Xiong ${ }^{1, \S}$ \\ I Institute of South China Karst, Guizhou Normal University, Guiyang, Guizhou 550001, China 2 Institute \\ of Entomology, Guizhou University, Guiyang, Guizhou 550025, China \\ † urn:lsid:zoobank.org:author:62E9B76A-24FF-4C61-9E33-E88393B5012A \\ ¥ urn:lsid:zoobank.org:author:9BA8A6EF-F7C3-41F8-AD7D-485FB93859F2 \\ § urn:lsid:zoobank.org:author:F3B9A06B-B8FC-4A29-9B7E-ODEEE2E3CF66 \\ Corresponding author: Kangning Xiong (xiongkn@163.com)
}

Academic editor: Michael Wilson | Received 14December 2010 | Accepted 1 February 2011 | Published 25 February 2011

urn:lsid:zoobank.org:pub:BD4409B1-B32E-4219-AAFA-49CA92B2D35E

Citation: Song Y, Li Z, Xiong K (2011) Three new species of the leafhopper genus Tautoneura Anufriev (Hemiptera, Cicadellidae, Typhlocybinae) from China. ZooKeys 83: 63-72. doi: 10.3897/zookeys.83.1178

\begin{abstract}
In the present paper, three new species are added to the genus Tautoneura Anufriev from China, T. baiyunshana sp. n., T. caoi sp. n. and T. yunnanensis sp. n. A key to species recorded from China is provided.
\end{abstract}

\section{Keywords}

Homoptera, Tautoneura, new species, morphology, taxonomy, China

\section{Introduction}

The leafhopper genus Tautoneura Anufriev (1969) belongs to the tribe Erythroneurini (Typhlocybinae) with Tautoneura tricolor Anufriev, 1969 as its type species. The genus consists of fifty-one species distributed in the Oriental and Palaearctic Regions. So far, twelve species in Tautoneura have been reported from China. A key to Chinese species 
and a species checklist of Tautoneura from China are provided. In this paper, three new species are described and illustrated from Henan, Yunnan and Gangsu Provinces of China. All specimens examined are deposited to the Institute of South China Karst, Guizhou Normal University (ISCK) and Insititute of Entomology, Guizhou University (IEGU), Guiyang, China.

\section{Materials and methods}

The specimens were obtained by sweep net method and were studied under Olympus SZX7 and CX41 microscopes. Morphological techniques and terminology follow Dietrich and Dmitriev (2006). Measurements of the new species are given in millimeters; body length is measured from the apex of head to apex of forewing in repose.

\section{Taxonomy}

\section{Tautoneura Anufriev, 1969}

Tautoneura Anufriev, 1969: 186-188

Erythroneura (Balia) Dworakowska, 1970 (Dworakowska, 1977: 290)

Erythroneura (Havelia) Ahmed, 1971 (Dworakowska, 1977: 290; Dworakowska 1980: 182)

Type species: Tautoneura tricolor Anufriev, 1969

Description. Body small, about 2.0-3.0 mm, usually yellow or light yellow. Head bluntly produced medially, slightly narrower than pronotum or equal to greatest width of pronotum. Median length of vertex equal to or longer than length between eyes. Some species with more rounded anterior margin of vertex. Pronotum broad, often with irregular patches or spots; scutellum nearly triangular. Forewings usually with red markings or spots.

Pygofer lobe broad, usually with several macrosetae at basal lower angle and some short stout setae in distal part of lobe on inner surface, peg-like. Dorsal appendage of pygofer long, tapering apically, movably articulated with pygofer lobe. Some species have ventral appendage. Aedeagus usually with large dorsal apodeme and one or two pairs of processes of variable length at apex of shaft. Style slender, with slim "neck" subapically, and prominent preapical lobe. Connective nearly M- or Y-shaped, central lobe well developed, as long as or little shorter than lateral arms. Shape of anal tube appendage diverse, but that of most species hook-like at apex.

Distribution. Palaearctic and Oriental Regions. 


\section{Key to males of Tautoneura from China}

1 Vertex with two longitudinal red stripes that converge at anterior margin midpoint of vertex ..................................................................... T. mori

- $\quad$ Vertex without convergent longitudinal stripes ............................................2

$2 \quad$ Aedeagal shaft without processes............................................................... 3

- $\quad$ Aedeagal shaft with one or more processes .................................................... 4

3 Aedeagus preatrium with one pair of processes........................... T. arachisi

- $\quad$ Aedeagus preatrium without processes (Figs 16, 17) .... T. yunnanensis sp.n.

$4 \quad$ Aedeagal shaft with two pairs of processes..................................................5

- $\quad$ Aedeagal shaft with only one or one pair of processes..................................

$5 \quad$ Aedeagal shaft process small, short, with one or more teeth .......................6

- $\quad$ Aedeagal shaft process long, slim, finger-like............................... T. formosa

6 Abdominal apodemes very slim, not extended beyond posterior margin of 3rd sternite (Fig. 3) .................................................. T. baiyunshana sp. $\mathbf{n}$.

- $\quad$ Abdominal apodemes expanded distinctly, extended to 5th sternite.....

T. longiprocessa

$7 \quad$ Aedeagal shaft with single, irregular process ................................... T. sinica

- $\quad$ Aedeagal shaft with pair of processes ...................................................... 8

$8 \quad$ Processes arising from apex or sub-apex of aedeagal shaft ...........................9

- $\quad$ Processes arising from base or sub-base of aedeagal shaft ...........................13

$9 \quad$ Processes arising from apex of aedeagal shaft ........................................10

- $\quad$ Processes arising from sub-apex of aedeagal shaft ............................. T. prima

10 Apex of style long and slim, slightly curved.................................... T. fusca Apex of style truncate or short and broad...............................................11

11 Pronotum with nearly rectangular red spot medially ........................ T. choui

- $\quad$ Pronotum without red medial spot ...................................................12

12 Forewing with three round red spots..................................... T. tripunctula Forewing with many orange-yellow markings, some areas with red spots or stripes

T. multimaculata

13 Aedeagus dorsal appendage bifurcate at apex (Fig. 22) ............ T. caoi sp. n. Aedeagus dorsal appendage not bifurcate at apex ......................... T. albida

(Note: the key does not include T. takaonella Mats. 1932, as only females of the species are known.)

Tautoneura baiyunshana Song, Li \& Xiong, sp. n.

urn:lsid:zoobank.org:act:87B96B3F-18A7-4F49-A9B4-B3D42863ECC4

Figures 1-9

Description. Body brownish yellow or brown testaceous. Head (Fig. 1) narrower than pronotum. Crown (Fig. 1) anteriorly produced medially, coronal suture distinct. Ver- 


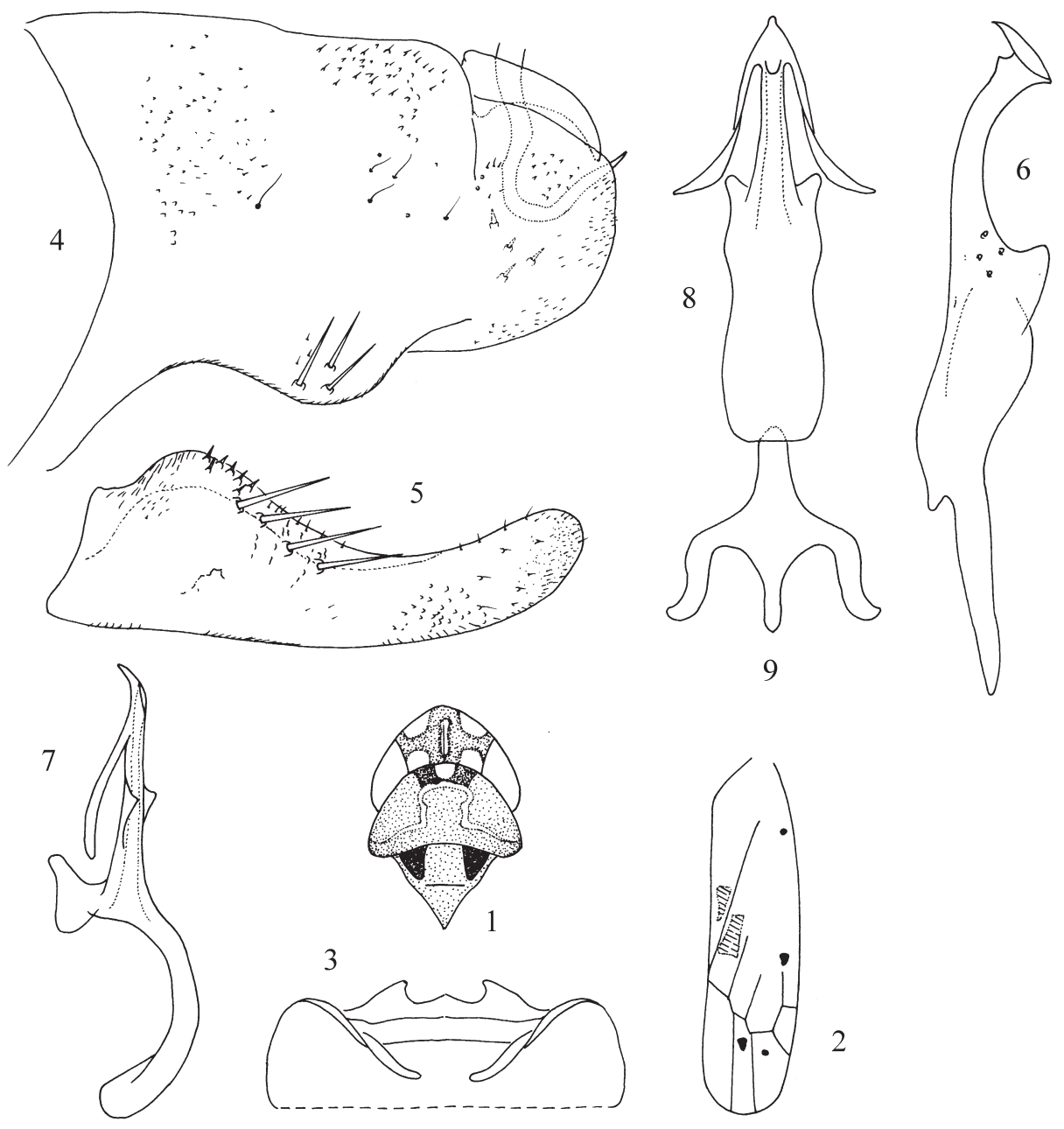

Figures I-9. Tautoneura baiyunshana Song, Li \& Xiong, sp. n. I Head and thorax, dorsal view 2 Forewing 3 Abdominal apodemes 4 Pygofer lobe, lateral view 5 Subgenital plate $\mathbf{6}$ Style 7 Aedeagus, lateral view 8 Aedeagus, ventral view 9 Connective.

tex (Fig. 1) median length little shorter than width between two eyes. Vertex and pronotum (Fig. 1) with milky yellow stripes. Eyes (Fig. 1) brownish yellow. Scutellum (Fig. 1) brownish yellow, basal triangles darker. Forewing (Fig. 2) brownish yellow, semitransparent, with four irregular dark spots and with two broad orange red patches near claval suture.

Abdominal apodemes (Fig. 3) small, not exceeding hind margin of 3rd sternite.

Pygofer (Fig. 4) broad, with three macrosetae at baso-lateral angle and a few sparse long fine setae. Pygofer microtrichia conspicuous, well developed. Pygofer dorsal appendage simple, not extended beyond pygofer apex, curved ventrally. Anal tube appendage (Fig. 4) hook-like apically. Subgenital plate (Fig. 5) lateral margin distinctly 
widened subbasally, with four macrosetae near median and with several short rigid setae along upper margin of sub-basal part. Style (Fig. 6) apex expanded, preapical lobe prominent. Connective (Fig. 9) nearly Y-shaped, central lobe and lateral arms slender; stem well developed, compressed. Aedeagal shaft (Fig. 7) almost straight, with pair of long processes arising near apex, another pair of short processes at middle area of shaft, triangular, lamellate in lateral view. Gonopore (Figs 7,8) subapical and on ventral margin. Dorsal apodeme (Fig. 7) short; preatrium (Figs 7, 8) long and slim, about as long as or little longer than shaft.

Measurement. Body length males 2.5 2.6 mm, females $2.6 \sim 2.8 \mathrm{~mm}$.

Host Plant. Unknown.

Type material. Holotype, male, China: Henan Province, Mt. Baiyunshan, 1300 1400 m, 17 August 2008, coll. Can Li. Paratypes: two males, ten females, same data as holotype.

Remarks. The new species is similar to Tautoneura longiprocessa Song \& Li (2008), but can be distinguished from the latter by the paired long processes arising from subapex of shaft (Figs 7, 8); gonopore (Figs 7, 8) subapical; preatrium (Fig. 7) slim, longer than aedeagal shaft.

Etymology. The new species is named after its type locality: "Baiyunshan", Henan Province.

\section{Tautoneura yunnanensis Song, Li \& Xiong, sp. n.} urn:lsid:zoobank.org:act:A8E8704B-EE04-457B-BA5E-1E00413FCDC7 Figures 10-18

Description. Body brownish yellow. Crown (Fig. 10) fore margin strongly produced and angulate medially. Coronal suture (Fig. 10) long, milky, nearly extended to 4/5 middle length of vertex. Two slim curving milky stripes situated at sides of coronal suture symmetrically. Eyes (Fig. 10) grey testaceous. Pronotum (Fig. 10) with two small orange red spots medially and broad area around them brownish. Scutellum (Fig. 10) light brown, with orange yellow spot at apex; basal triangles orange yellow. Forewing (Fig. 11) with four orange markings around claval suture and several brownish or blackish brown spots.

Abdominal apodemes (Fig. 12) large, broad, reaching 5th sternite.

Pygofer lobe (Fig. 13) broad, with two macrosetae at basal lower angle and numerous fine setae or microsetae distributed on lateral surface. Pygofer microtrichia distinct. Pygofer dorsal appendage expanded at base and tapering towards apex, bent ventrally. Anal tube appendage (Fig. 13) slim, hook-like apically. Subgenital plate (Fig. 14) with three basal macrosetae, expanded subbasally and with several peg-like short setae. Style (Fig. 15) quite long, apex expanded obviously; preapical lobe large, prominent. Connective (Fig. 18) Y-shaped, lateral arms strong, central lobe and stem well developed. Aedeagal shaft (Figs 16, 17) almost straight and short in lateral view, its base conspicuously expanded in ventral view; without any process. Gonopore (Figs 16, 17) broad, 

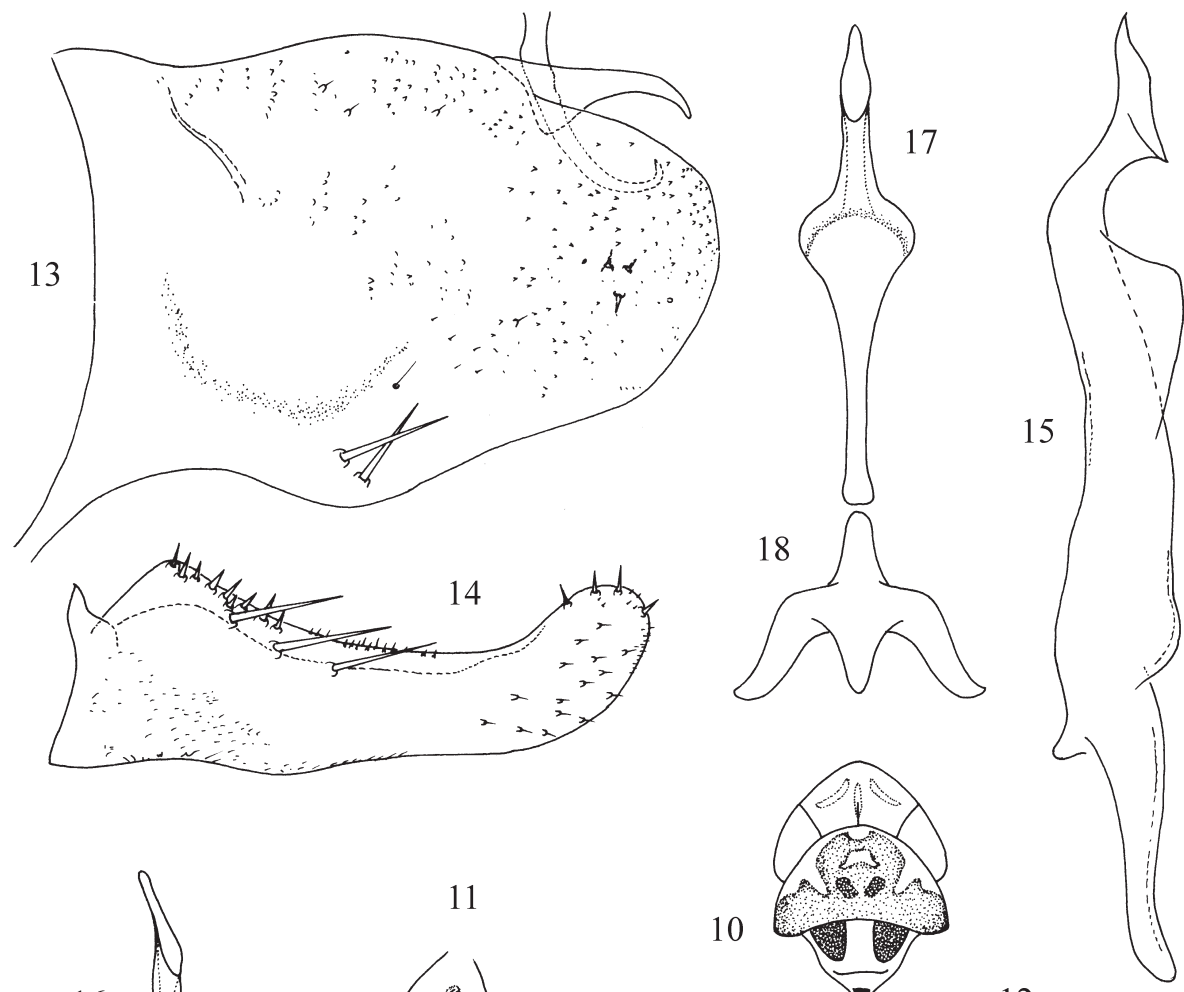

16

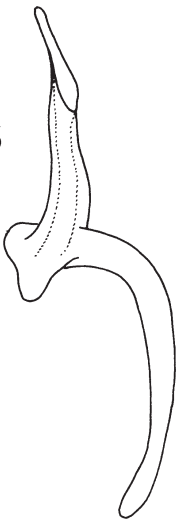

11

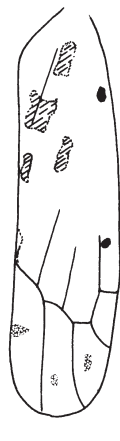

10

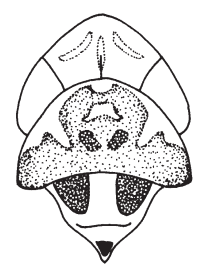

12

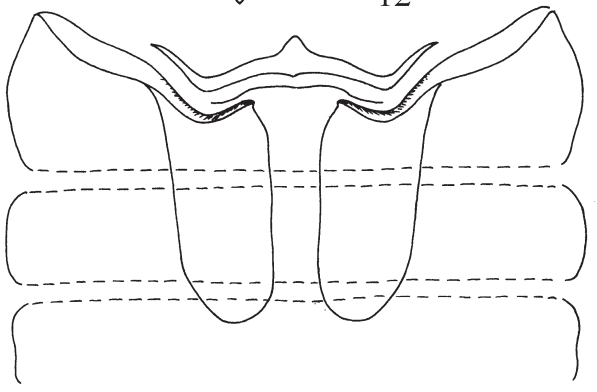

Figures 10-18. Tautoneura yunnanensis Song, Li \& Xiong, sp. n. 10 Head and thorax, dorsal view I I Forewing $\mathbf{2}$ Abdominal apodemes $\mathbf{3}$ Pygofer lobe, lateral view I4 Subgenital plate I5 Style $\mathbf{6}$ Aedeagus, lateral view 17 Aedeagus, ventral view 18 Connective.

apically. Aedeagus dorsal apodeme (Fig. 16) short and small; preatrium (Figs 16, 17) much longer than aedeagal shaft.

Measurement. Body length males 2.2 2.3 mm.

Host plant. Unknown.

Type material. Holotype, male, China: Yunnan Province, Lijiang, 16 July 2010, coll. CAN LI. Paratypes: one male, same data as holotype; one male, China: Yunnan Province, Mengla County, 18 July 2008, coll. YUEHUA SONG. 
Remarks. The new species is similar to Tautoneura misrai Dworakowska (1977), but can be distinguished from the latter by the large and broad, apical gonopore; the aedeagus dorsal apodeme short, not expanded distinctly and the ventral appendage absent; the forewing with four orange patches near claval suture.

Etymology. The new species is named after its type locality: "Yunnan", China.

\section{Tautoneura caoi Song, Li \& Xiong, sp. n.}

urn:lsid:zoobank.org:act:1CAE0426-23ED-4C0B-AC2A-7013DBAC940F

Figures 19-27

Description. Body yellowish. Structural characters as in T. baiyunshana sp. n. and T. yunnanensis sp. n. Vertex and pronotum (Fig. 19) with irregular orange red markings. Eyes grey. Scutellum (Fig. 19) basal triangles orange yellow and apex with dark spot. Forewing (Fig. 20) brownish yellow, semitransparent, with numerous orange yellow markings, some parts with red spots or streak, apex (apical cells) dark brown.

Abdominal apodemes (Fig. 21) broad, extended beyond posterior margin of 3rd sternite.

Pygofer lobe (Fig. 22) broad, with numerous macrosetae, long fine setae and rigid short setae. Pygofer microtrichia inconspicuous. Dorsal appendage bifurcate far from base, extended beyond pygofer apex. Subgenital plate (Fig. 23) with four basal macrosetae and distinct marginal subbasal rigid setae formed continuous row. Style (Fig. 24) slender, apex expended slightly; preapical lobe prominent. Connective (Fig. 27) Y-shaped, two arms strong, central lobe well developed. Aedeagal shaft (Figs 25, 26) short, with pair of lateral processes at sub-base, part between aedeagal shaft and preatrium expanded. Gonopore nearly median, on ventral margin. Dorsal apodeme little longer than that of other two new species. Preatrium long, much longer than aedeagal shaft.

Measurement. Body length males 2.5 2.6 mm, females 2.7 2.8 mm.

Host plant. Ulmus pumila L. (Elm)

Type material. Holotype, male, China: Gansu Province, Zhenyuan County, 19 May 2010, coll. WEI CAO. Paratypes: seven males, ten females, same data as holotype.

Remarks. The new species is distinguishable from T. ahmedi Dworakowska (1977) by the aedeagus preatrium strongly expended at terminal part; the dorsal appengage bifurcate apically and the gonopore near median area of aedeagal shaft.

Etymology. This sepcies is named after its collector.

\section{Species checklist of Tautoneura}

T. (Havelia) ahmedi Dworakowska 1977. India

T. (Havelia) alba (Ahmed, 1971) India

T. (T.) albida (Dworakowska 1970). China (Guangdong) 


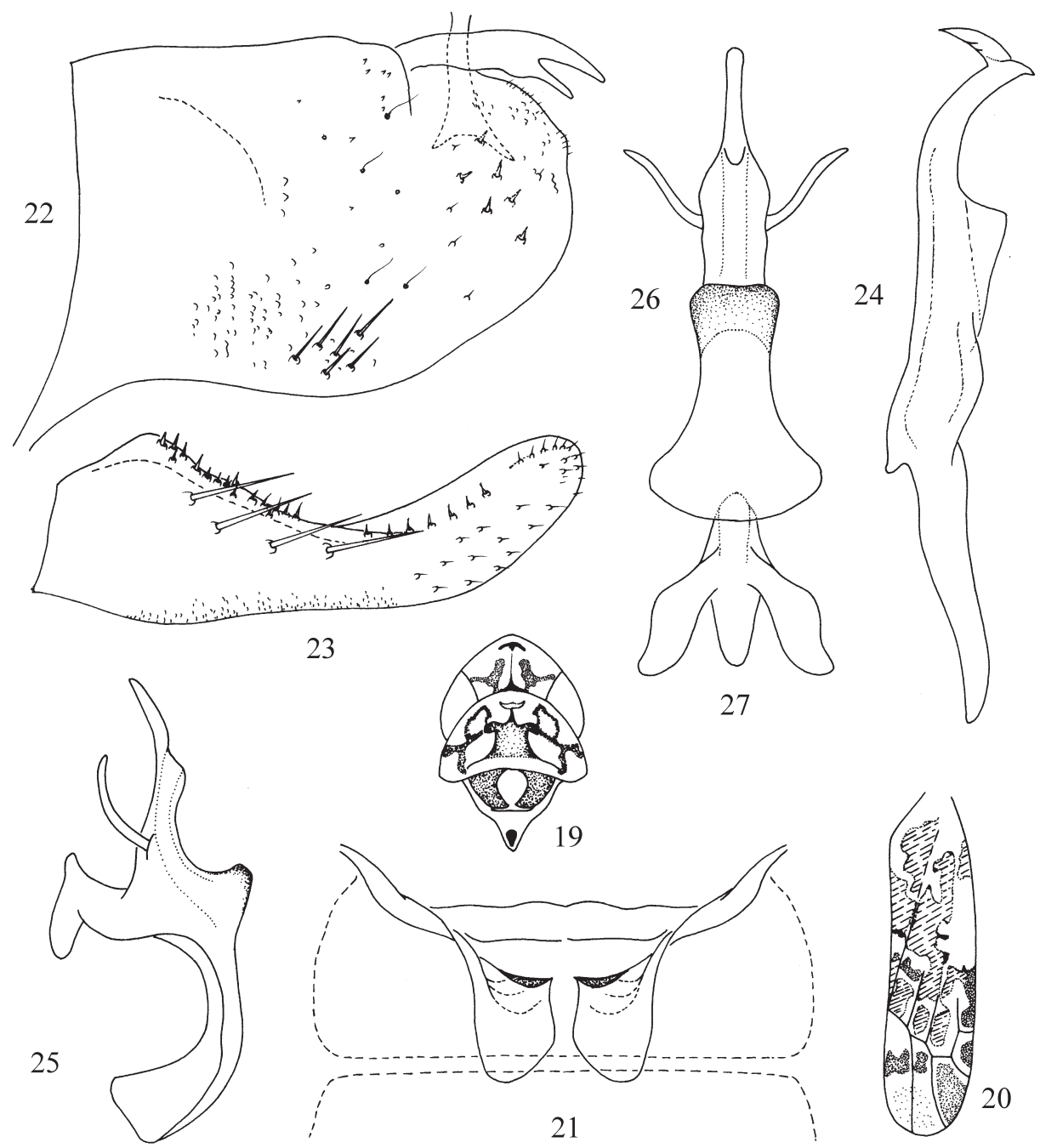

Figures 19-27. Tautoneura caoi Song, Li \& Xiong, sp. n. 19 Head and thorax, dorsal view 20 Forewing 21 Abdominal apodemes 22 Pygofer lobe, lateral view $\mathbf{2 3}$ Subgenital plate $\mathbf{2 4}$ Style $\mathbf{2 5}$ Aedeagus, lateral view $\mathbf{2 6}$ Aedeagus, ventral view $\mathbf{2 7}$ Connective.

T. (T.) arachisi (Matsumura, 1916). China (Taiwan)

T. (Havelia) bellula Dworakowska 1994. Sikkim

T. (T.) bena Dworakowska 1981 India

T. (Havelia) baiyunshana Song, Li \& Xiong, sp. n. China (Henan)

T. (Havelia) caoi Song, Li \& Xiong, sp. n. China (Gansu)

T. (Havelia) choui Ma 1983. China (Shaanxi)

T. (T.) deska (Dworakowska 1970). Samoa

T. (T.) dubiosa Dworakowska 1981. Nepal

T. (T.) dubiosissima Dworakowska 1981. Nepal 
T. (T.) dukara Dworakowska 1981. India

T. (T.) eda Dworakowska 1981. India

T. (T.) erythropunctata (Ramakrishnan \& Menon, 1973) India

T. (T.) ficaria Dworakowska, 1984. India; Singapore

T. (T.) formosa (Dworakowska 1970). China (Jiangsu)

T. (T.) fusca (Dworakowska 1970). China (Guangdong)

T. (T.) incisa Dworakowska 1980. India

T. (T.) indefinita (Dworakowska 1970). Samoa

T. (T.) japonica Dworakowska, 1972. Japan

T. (T.) kira Dworakowska 1981. India

T. (T.) klara Dworakowska 1981. India

T. (T.) leucothoe (Kirkaldy, 1907). Fiji; Samoa

T. (T.) longiprocessa Song and Li 2008. China (Guizhou)

T. (Havelia) maculosa Sohi, Mann \& Shenhmar, 1987. India

T. (Havelia) manica Thapa, 1989. Nepal

T. (T.) marthae (Linnavuori, 1960). Fiji

T. (T.) mayarami Mathew \& Ramakrishnan, 1996. India

T. (T.) misrai Dworakowska 1977. India

T. (T.) mori (Matsumura, 1910). China (Shangdong, Anhui, Jiangsu, Zhejiang, Sichuan, Guizhou)

T. (T.) mukla Dworakowska 1981. India

T. (Havelia) multimaculata Song and Li, 2009. China (Guizhou).

T. (T.) mureda Dworakowska 1981. Nepal

T. (T.) napa Dworakowska 1981. India

T. (T.) ochreleuca Thapa, 1984. Nepal

T. (Havelia) panthera Dworakowska 1994. Sikkim

T. (T.) panti Dworakowska, 1977. India

T. (Havelia) pewna Sohi \& Mann, 1992. Nepal

T. (T.) prima Dworakowska 1979. China (Guizhou)

T. (T.) redama Dworakowska 1981. Nepal

T. (T.) sanguinalis (Distant, 1918). India

T. (T.) secunda Dworakowska 1979. Vietnam

T. (T.) sinica (Dworakowska 1970). China (Guangdong, Jiangsu)

T. (T.) smocza Dworakowska 1980. India

T. (T.) takaonella (Matsumura 1932). China (Taiwan)

T. (Havelia) tricolor Anufriev 1969. Russia

T. (T.) tripunctula (Melichar, 1903). China (Guizhou, Yunnan)

T. (Havelia) unicolor Dworakowska 1979. Vietnam

T. (Havelia) yunnanensis Song, Li \& Xiong, sp. n. China (Yunnan)

T. (T.) zembata Dworakowska 1979. Japan

T. (T.) zizypha Thapa, 1984. Nepal

T. (T.) zobra Dworakowska 1979. Vietnam

T. (Havelia) zygina Dworakowska 1994. Sikkim 


\section{Acknowledgements}

We would like to express our sincere thanks to Mr. Wei Cao, Bureau of Agriculture and Pasture of Zhenyuan County, Gansu, China, for providing material for the study and Dr. Can Li, Department of Biology and Engineering of Environment, Guiyang University, China for help in the current research. The present study is part of a wider survey of Typhlocybine leafhoppers from China. The project was supported by the Guizhou Provincial Natural Science Foundation of China (No. [2010]2063) and Key Technologies R\&D Program of Guizhou Province (SY[2010]3068).

\section{References}

Anufriev GA (1969) New and little known leaf-hoppers of the subfamily Typhlocybinae from the Soviet Maritime Territory (Homoptera, Auchenorrhyncha). Acta Faunistica Entomologica Musei Nationalis Pragae 13: 186-188.

Dietrich CH, Dmitriev DA (2006) Review of the New World genera of the leafhopper tribe Erythroneurini (Hemiptera: Cicadellidae: Typhlocycbinae). Illinois Natural History Survey Bulletin 37(5): 119-190.

Dworakowska I (1970) A new subgenus of Erythroneura Fitch (Auchenorrhyncha, Cicadellidae, Typhlocybinae). Bulletin de l'Academie Polonaise des Sciences, Serie des Sciences Biologiques 18(6): 347-354.

Dworakowska I (1977) On some north Indian Typhlocybinae (Homoptera, Auchenorrhyncha: Cicadellidae). Reichenbachia 16(29): 290-293.

Dworakowska I (1979) On some Erythroneurini from Vietnam (Typhlocybinae, Cicadellidae). Annotationes Zoologicae et Botanicae 131: 27-28.

Dworakowska I (1980) On some Typhlocybinae from India (Homoptera, Auchenorrhyncha, Cicadellidae). Entomologische Abhandlungen und Berichte aus dem Staatlichen Museum fur Tierkunde in Dresden 43: 151-201.

Dworakowska I (1981) Proskura gen. n. and some Erythroneurini from Souther India (Homoptera, Auchenorrhyncha, Cicadellidae, Typhlocybinae). Reichenbachia 19(37): 231, 241.

Dworakowska I (1994) Typhlocybinae (Auchenorrhyncha, Cicadellidae) known to occur in Sri Lanka. Annotationes Zoologicae et Botanicae 216: 30-33.

$\mathrm{Ma}$ (1983) A new species of the genus Tautoneura and a new record from China. Entomotaxonomia 5(2): 149-150.

Matsumura S (1932) A revision of the Palaearctic and Oriental Typhlocybid-genera with descriptions of new species and new genera. Insecta Matsumurana 6(3): 118.

Song YH, Li ZZ (2008) Notes on Chinese species of the genus Tautoneura Anufriev (Hemiptera: Cicadellidae: Typhlocybinae), with descriptions of two new species. Zootaxa 1769: $60-68$. 